\title{
Theses on Biosemiotics: Prolegomena to a Theoretical Biology
}

\author{
Kalevi Kull \\ Department of Semiotics \\ University of Tartu \\ Tartu, Estonia \\ kalevi.kull@ut.ee \\ Terrence Deacon \\ Department of Anthropology \\ University of California, Berkeley \\ Berkeley, CA, USA \\ deacon@berkeley.edu

\section{Claus Emmeche} \\ Center for the Philosophy of Nature \\ and Science Studies \\ University of Copenhagen \\ Copenhagen, Denmark \\ emmeche@nbi.dk

\section{Jesper Hoffmeyer} \\ Department of Molecular Biology \\ University of Copenhagen \\ Copenhagen, Denmark \\ jhoffmeyer@mail.dk

\section{Frederik Stjernfelt} \\ Center for Semiotics \\ Aarhus University \\ Aarhus, Denmark \\ semfelt@hum.au.dk
}

\begin{abstract}
Theses on the semiotic study of life as presented here provide a collectively formulated set of statements on what biology needs to be focused on in order to describe life as a process based on semiosis, or signaction. An aim of the biosemiotic approach is to explain how life evolves through all varieties of forms of communication and signification (including cellular adaptive behavior, animal communication, and human intellect) and to provide tools for grounding sign theories. We introduce the concept of semiotic threshold zone and analyze the concepts of semiosis, function, umwelt, and the like as the basic concepts for theoretical biology.
\end{abstract}

\section{Keywords}

biosemiotics, communication, function, relations, semiosis, semiotic threshold zone, signification, umwelt 
The variety of scientific disciplines that constitute modern biology and semiotics, which is the science of sign systems, commonly known from the study of human language and social-sign systems, have recently demonstrated trends toward a recognition that sign processes per se and the processes of life may be intimately and inseparably interconnected. This view has developed into a general approach called biosemiotics. The term "biosemiotics" appears to have been coined by Friedrich S. Rothschild in 1962, but Thomas Sebeok played a major role in defining the field in the 1980s and 1990s (see, e.g., Anderson et al. 1984; Sebeok and Umiker-Sebeok 1992; Sebeok 1996, 2001). ${ }^{1}$

There have been several attempts to formulate the basic principles of biosemiotics, in both extensive versions (Hoffmeyer 1996, 2008) and the more compact ones (Sebeok 1996; Hoffmeyer 1997; Emmeche et al. 2002; Stjernfelt 2002), together with detailed analyses of some central biosemiotic problems (Deacon 1997; Kull et al. 2008). Still, the establishment of biosemiotics requires, on the one hand, a deepening and grounding of the theory of semiotics and, on the other hand, a development of a richer theoretical biology. But as those of us who identify as biosemioticians have begun to organize international associations, annual meetings, edited volumes, and journals devoted to this new field, it has become apparent that a single well-defined paradigm is still in the process of coalescing from a diverse collection of theoretical positions. Although such diversity is a healthy starting point from which to develop an intellectually productive field of research, it is also important to develop a clear sense of the scope of these various visions of the field. To accomplish this it is necessary to first identify points of common terminology and shared theoretical assumptions and then to identify incompatible frameworks and conceptual issues that still need to be resolved. This article represents an effort to articulate a common set of assumptions that are shared among a group of researchers in the field, who ground their work on a strongly Peircean framework. It is hoped that carefully outlining and analyzing our shared theoretical assumptions will help clarify and contrast this approach with respect to others.

Because a significant number of those of us who identify themselves as Peircean biosemioticians are located in northern Europe, we agreed to meet and attempt to formulate such a document during August 1-6, 2008, at a meeting in Estonia (at Saka cliff on the coast of the Baltic Sea) by the invitation of Tartu semioticians. Our aim was to formulate a joint understanding of the conceptual basis and the basic principles of a semiotic study of life, i.e., biosemiotics. The results are arranged in the form of the eight theses that follow.

\section{Eight Theses}

(1) The semiosic-non-semiosic distinction is coextensive with the life-nonlife distinction, i.e., with the domain of general biology.

The concepts of function and semiosis (sign processes) are intertwined. Both are teleological concepts in the sense of being determined with respect to an end (or other than itself) - a specifically correlated absent content. Although it is unclear whether these two properties of living processes (function and semiosis) are exactly coextensive, it is clear that although time-asymmetrical, irreversible physical processes are found in the prebiotic physicochemical world; teleological processes that are specially organized with respect to specific ends or referents are unique to living processes.

If we think of a function as a process organized around an implicitly represented end, then these two classes of phenomena must be considered entirely coextensive. Alternatively, semiosis, the activity of sign processes, may be considered only in conditions under which there is explicit or implicit representation of an end state or under which a functional satisfaction condition can be identified as holding or not holding, in which case semiosis can be defined with respect to prior function.

With the demonstration of the plausibility of proto-life processes such as autocells (Deacon 2006a), which are recursively self-maintenant and so can self-repair, reproduce, and evolve, it becomes difficult to identify the exact threshold of the onset of semiosis, and yet function can be unambiguously demonstrated by the presence of the component processes. This, then, allows us to identify a lower limit to the identification of functional organization-thus telos-even though explicit semiosis is still ambiguous. This identifies a threshold zone below which semiosis is not defined but above which there can be stages of semiotic differentiation. It is thus an open and crucial issue of research to determine, empirically and conceptually, the different thresholds in this zone between such simple reproducing and evolving systems and contemporary terrestrial organisms that appear to depend unambiguously on semiotic processes.

Often the emergence of life is seen as a sudden transition where the many properties defining life arise together or are tightly interconnected (like self-replication, autocatalysis, function, and cellularity). However, this appears to be both too simple and implausible. There is no simple dividing line where all the interconnected properties of living systems, as we know them, emerge. Instead we observe what we call a threshold zone, probably involving incremental stages in which different component processes emerge. This is an open issue for further investigation and will probably develop into a fertile area for both molecular biology and biosemiotic research to contribute. 
(2) Biology is incomplete as a science in the absence of explicit semiotic grounding.

Neo-Darwinian biology as practiced all over the world has prescinded (i.e., abstracted from necessary contextual support) an asemiotic conception of life as mere molecular chemistry, and yet at the same time it is dependent on unanalyzed semiotic assumptions. The reason why this is not felt as a problem is that biology compensates for the excluded semiosis by introducing a plethora of implicitly semiotic terms like "information," "adaptation," "signal," "cue," "code," "messenger," "fidelity," and "cross talk." These uses are seldom well defined and are often applied in an allegedly metaphoric way, with the implicit assumption that they can be reduced to mere chemical accounts if necessary.

It is not clear, however, that a complete and unproblematic reduction of this sort is possible. If biologists were asked to avoid these implicitly semiotic terms they would have a hard - and probably impossible - job of explaining the nature of organic function. For example, if hemoglobin were known only by its three-dimensional molecular structure, it would not be possible to guess that it functioned as a transporter for oxygen. But knowing that hemoglobin is a reflection of the need of multicellular organisms to provide energy for the metabolism of somatic tissues, it immediately becomes clear (1) that it must have some structural features conducive to binding and transporting oxygen in blood, (2) that the oxygenbinding region of the hemoglobin molecule is expected to be conserved throughout evolution, and (3) that different forms of hemoglobin differ in specific ways that correspond to different oxygen transport requirements (e.g., in different species or in mammalian gestation).

The theoretical issue at stake here is that in biology empirical facts are always contextually constrained. Contextuality should not be conceived as a free ticket to determinations from outside domains-rather, contextuality is constrained by function (and vice versa). If life exists on distant planets in other regions of the universe with large heterotrophic forms, it is likely that they will also require a corresponding transporter molecule, and if their metabolism is mediated by oxygen, then the transporter molecules might not resemble our Earth-hemoglobin in narrow molecular detail but would nonetheless retain the capacity to carry oxygen in a similarly protected way so that it can be released again in tissues in need of it. And if their metabolism is mediated by a substance other than oxygen, they will still require some substance undertaking the analogous function of facilitating energy transport. This already puts constraints upon the space of possible realizations of the "hemoglobin molecule" (or any such nonterrestrial functional analog), which severely restricts its form and its correspondence to contextual factors.
Another way to put this is to say that the function of hemoglobin is not intrinsic to its molecular structure. Rather it is relational-hemoglobin may be seen as a carrier of constitutive absence (Deacon 2006b), in the sense that the molecule's properties are constituted not only by intrinsic features but also by extrinsic features of its historical and physical functional contexts. In effect, the missing oxygen with respect to which hemoglobin structure has evolved has become its defining characteristic. In this respect, one can understand the structure of hemoglobin as a "representation" of both oxygen and its role in the cellular molecular processes of metabolism. The function of hemoglobin is in this way what affords the possibility of it having representational character. This function relates to the "needs" or self-maintenance conditions of some agent. "Needing something" implies both its transient absence and some structure or processual state representing that absence and its possible ending or completion.

This constitution with respect to something extrinsic and/or absent shows that function and representation are two aspects of the same mode of relational existence. This implies that the primary unit of biosemiotic research is a sign-not merely a molecule or cell.

(3) The predictive power of biology is embedded in the functional aspect and cannot be based on chemistry alone.

It is an accepted truth in biology that structure and function are interdependent; e.g., a biological explanation is incomplete even if the production and the structure of a macromolecule in a cell have been exhaustively described. There is a missing feature of the explanation-we still need an answer to the question "What is it for?" Answering this question is part of the functional contextualization that all biological facts require. In many cases, these functions are characterized as being of a regulatory, information-carrying, or signaling kind, and thus describing the function of these structures is really embedding them in a wider system that has a sign-processing (e.g., signal-transducing) character. In this wider system, the functions that a macromolecule take part in (or contribute to) can be fulfilled by other slightly or completely different structures (say, being only similar regarding an "active site"), and thus, the structure is seen as a vehicle for fulfilling that function. Locating and clarifying functional-processual parts in a wider network within metabolism enables partial predictions of some constraints that need to be met if the vehicle of a function should be able to work. Most of the predictive power of biology is lost if semiofunctional analysis is excluded.

The difficulty of making predictions about biological phenomena is that the functions are plurally realizable and thus subject to considerable variation. As a result, the physicochemical details necessarily provide an incomplete account. 
Functional requirements do, however, constrain the physicochemical substrates that can be recruited.

\section{(4) Differences in methodology distinguish a semiotic biology} from non-semiotic biology.

It is the aim of biosemiotics to make explicit those assumptions that are imported into biology by such unanalyzed teleological concepts as "function," "information," "code," "signal," and "cue" and to provide a theoretical grounding for these concepts. The widespread use of such terms in existing biology points to the fact that such notions cannot be avoided or fully substituted with merely chemical accounts. Biosemiotics has the scientific task of (1) grounding such terms in a physicobiological context, (2) defining and interrelating such terms with the constant aim of avoiding the anthropomorphisms that threaten when they are left with implicit definitions only, and (3) so to make biology theoretically complete.

Kant's Kritik der Urteilskraft, one of the early masterpieces of theoretical biology, provides some interesting definitions of teleology in biology. Kant has described organisms as possessing a "formative power" to construct themselves as an "organized and self-organized being" in which "every part is reciprocally both ends and means" (Kant 1790: Part II, paragraph 66; see also Stjernfelt 2007: 1999ff.). It is important to underline that in making these definitions of teleology, Kant is, as so often, criticizing naive metaphysics - where the idea of a telos is treated as something external to nature, which by means of strange, unknown powers affects natural processes. In contrast, his definition of telos is functional and thus internal to nature and characterizes a specific class of natural processes. It is a concept of telos that does not refer to unknown vitalist forces but rather defines telos by a specific class of causal processes.

Modern biology has been working on the assumption that there is an incompatibility between the teleological and physicochemical characterizations of life. Biosemiotic approaches assume that there is no deep incompatibility and that a principled theory unifying these domains is possible.

(5) Function is intrinsically related to organization, signification, and the concept of an autonomous agent or self.

Functions are not only the output of evolutionary history; rather, functionality is the prerequisite for organic evolution. For instance, autocells do not in all cases have an evolutionary history, but they do have functions. Evolution presupposes function rather than vice versa. Natural selection cannot be defined except with respect to a bounded, self-maintaining, and self-reproducing dynamical unit system. A discrete system with these properties must therefore be constituted by component materials and dynamical processes that reciprocally generate each other as well as their collective organization. The critical features and dynamical actions of these components exemplify Kant's criteria for possessing intrinsic telos and are thus functional. The possibility of evolution derives from the fact that functions, because they can be realized multiple number of times, can coopt any incidental physicochemical properties of the substrates they utilize. Likewise, semiosis can coopt any incidental feature exhibited by functional processes or their properties.

An unresolved question arises with respect to the relationship between semiosis and function and as to which terminological paradigms can best characterize this fundamental relationship. Thus, the coupling of perception signs and action signs in Uexküll's functional cycle (Uexküll 1921; Kull 2001) has an "if-then" inference structure. ${ }^{2}$ Perception signs form the premises of the conclusion inherent in the ensuing action signs. Perception signs grant (with some probability) that something is the case, and consequently, the organism "makes a decision" to act on the basis of this information. This functional cycle thus has an if-then structure, like a fallible inference of an inductive, a deductive, or an abductive form. However, defining functional and semiotic processes by reference to the concept of inference risks the charge of circularity. To avoid confusion, other terms might be preferred, e.g., conditional causality and proto-argument.

Peirce identified semiotics with logic in the broadest sense. As we implied above, adaptation also involves the selective semiotic recruitment of those physicochemical aspects of the organism environment that are relevant to the persistence of that process. As a consequence, conditional relationships of logic become represented in the forms and habits of organisms and their components embodying this "bio-logic." In this way, semiosis facilitates the development of an organism's capacity to behave in a way that is both consistent with its environment and implicitly inferential. "Logic" as we are using it here is not something to be considered as a product of abstract cognition in humans, but rather we simply intend to highlight the inference-like architecture of biological function, which we take to also be the basis of semiosis in general.

\section{(6) The grounding of general semiotics has to use biosemiotic} tools.

Biosemiotics does not take for granted the wide variety of concepts of the sign, sign action, and so on in the different semiotic traditions but undogmatically sees these as a resource for the construction of an up-to-date, refined, and better-grounded (as concerns contemporary biology) version of a general semiotics. The aim is to understand the dynamics of organic mechanisms for the emergence of semiotic functions, in a way that is compatible with the findings of contemporary biology and yet also reflects the developmental and evolutionary history of sign functions.

What do signs do? They stabilize or secure reliable modes of self-maintenance in such a way that they are able to expand 
the realm of processes that have already proved functional in the past. ${ }^{3}$ They do this in an economical way, allowing the recognition of no more than an aspect of an object to suffice for the organism to act upon that object. Of course, the flipside of this economy is fallibility, but fallibility also provides the space of alternatives that makes evolution possible. This securing of prior forms and dynamical relationships implies "remembering" what has already proved functional for selfmaintenance. Remembering a bio-form is like remembering its recipe for its way of production or regeneration. ${ }^{4}$ To remember in this biological sense is to be able (for some sign system) to put to use that set of constraints or imposition of boundary conditions that confine physical and chemical processes to actualize the means of production of these same forms. ${ }^{5}$

\section{(7) Semiosis is a central concept for biology that requires a more exact definition.}

Although there are many descriptions of semiotic processes, it is still an unresolved challenge to provide an account that explains what exactly constitutes semiosis without either assuming a homuncular interpreter or leaving critical relationships undefined. While this is not so problematic for human or complex animal communication, where an interpreter can be provisionally assumed without further explanation, it becomes a serious challenge for fundamental issues in biosemiotics, since we cannot in these cases appeal to an extrinsic interpreter. The organism (or the organism plus its environment) must, in itself, constitute an interpreter, but in biosemiotic analysis we must attempt to be explicit in explaining specifically which processes provide the necessary and sufficient conditions to consider that process semiosis.

The interpretive capacity is an emergent property of a reciprocal end-means relationship of a self-propagating dynamical system. The constitutive absence (Deacon 2006b, discussed above) is the basis of both biological function to and dependence on an environment. Because an organism must incessantly remake itself, utilizing resources afforded by its environment, it must be in dynamical correspondence with these crucial intrinsically absent features, and at the same time its constituent parts and dynamics must be reciprocally generating one another with respect to this absence. In this respect, an organism is a sign-interpreting process that can be described as a recursive self-referential sign production process, dependent on or influenced by some external factors likely to be present in its environment.

We are not currently in a position to provide a more precise and unambiguous description of the interpretive architecture that is implicit in an organism. However, we can identify many critical component processes and relationships that must be involved, and we can provide a rough sketch of what the simplest model of the creation of a semiotic relationship should involve.
We can identify seven properties or conditions that must be met. The following is a rough sketch of these critical conditions:

(a) Agency. A unit system with the capacity to generate enddirected behaviors.

(b) Normativity. A semiotic process builds up normative properties in a broad sense, thus being itself embedded in a process that contributes normativity. This includes the possibility that the representation is in error or that its consequence (in Peircean terms, its dynamical interpretant) can be either compatible with or incompatible with preserving the integrity of the living system in which it occurs.

To grasp this minimal notion of normativity, think of the difference between a physical pattern as such and that pattern serving a function. Any specific physical pattern may be characterized by algorithmic information theory as highly random, or highly regular, or something complex in between, be it either descriptively compressible or truly complex and incompressible. But a pattern serving a function has, in addition to its own high or low algorithmic information content, a degree to which it serves, or fails to serve, its goal. For semiotic processes (having such functions as representation, information storing, and interpretation) the degree to which a pattern serves or fails to serve such functions constitutes a norm.

(c) Teleo-functionality. Semiosis is always embedded in a process that is end directed in which the semiosis can be assessed with respect to whether its interpretation is concordant or discordant with the dynamics of achieving that end. This is what determines the normative properties of a sign-interpreting process.

(d) Form generation. The systemic organization that is responsible for interpreting the semiotic function of a sign vehicle must include a form-generating process that directly or indirectly contributes to the persistence (re-presentation) of that function. The interpretation process is constituted by generating a structure (physical form) that serves as a sign of the prior sign and also can produce further structural consequences.

(e) Differentiation of a sign vehicle from the dynamics of the reciprocal form-generating process. A sign vehicle must be insulated from the dynamics that it constrains and that is responsible for generating a repetition of this process. ${ }^{6}$

(f) Categorization. Sign repetition can never be $100 \%$ physically identical. This, together with normativity, is why signs form types. Categorization appears in all communication processes in case of adaptive-enough systems. Functionally similar instances of signs (tokens) are subsumed under a general type. At the same time, what signs refer to is also categorized. This aspect of the use of signs is highly economical because it enables the organism to get by with the generation of only a finite number of simple, typical modes of interpretive action 
to achieve similar ends. The flip side of this is, of course, the possibility of fallacy (cf. the discussion above).

(g) Inheritance of relations. Various developmental processes include those that have created novel fitted correspondence relationships among the parts of the organism and among the organism and its environment, and are presumed to be the principal means by which semiotic relationships are generated. In this respect, genetic inheritance represents one of the most basic forms of semiosis, and so studying the conditions of its generation should provide insight into the way semiotic process becomes grounded in physical processes. Besides genetic inheritance, there are several other forms of inheritance (e.g., epigenetic, neural, and social) that are in use by various communication processes. In this sense, semiotic processes include memory processes in general, which maintain continuity of information and stability of dynamical options.

Thus, aboutness can exist without invoking mental (sensu stricto) operations (processes taking place in brains, possibly involving, say, awareness or consciousness). Conversely, mental operations in this sense may evolve as a higher-order augmentation of the capacities to generate and process aboutness.

\section{(8) Organisms create their umwelten.}

Organisms, as embodiments of semioses, are not separable from the environment without loosing their essential nature. Therefore, a number of specific concepts that describe these relationships are essential.

The umwelt is the set of features of the environment as distinguished by the organism, or the self-centered world that relates an organism with the else. This concept was introduced into biology by Jakob von Uexküll $(1921,1982)$ and has become widely used and has been further developed in semiotics, anthropology, philosophy, and other fields, especially since late 1970s.

A semiotic niche is defined as the totality of signs or cues in the surroundings of an organism-signs that it must be able to meaningfully interpret to ensure its balance and welfare. The semiotic niche includes the traditional ecological niche factors, but now the semiotic dimension of these factors is also emphasized. The organism must distinguish relevant from irrelevant food items and threats, for example, and it must identify the necessary markers of the biotic and abiotic resources it needs, namely, water, shelter, nest-building materials, and mating partners. The semiotic niche thus comprises all the interpretive challenges that the ecological niche forces upon a species.

The semiotic niche in this way may be seen as an externalistic counterpart to the umwelt concept: if the umwelt denotes an internal model in the organism, then the semiotic niche refers to a segment of the external environment. It makes the umwelt concept compatible with an evolutionary approach, since now one may pose the question of whether the umwelt of a species is sufficiently differentiated to meet the challenges posed by the available semiotic-niche conditions.

\section{Conclusions}

Biosemiotics sees itself as an extended and more general approach to biological explanation that complements and augments the concept of biological function. Thus the physicochemical account of biological phenomena can be seen as a special case within biosemiotics. Likewise, this concerns the different branches and theories in biology, for instance, the neo-Darwinian theory of evolution becomes a special case of a biosemiotic theory of evolution.

In relation to semiotics, biosemiotics provides a way for grounding its theory. To the extent that semiotic theories of social and communicative processes at the human scale are defined with respect to human or animal interpreters and thus defined indirectly with respect to the biological processes constituting minds, most semiotic theories must implicitly appeal to a biosemiotic interpretation process.

Recognizing this necessary dependence is the first step toward understanding how the humanities and sciences might be integrated into a new grand synthetic theory without having to reduce one to the other.

\section{Notes}

1. On the history of biosemiotics, see Kull (1999) and Favareau (2007).

2. This might align it with Peirce's notion of an argument; "argument" here is of course not taken in the sense of symbolic logic where all internal structure of it needs to be explicit but in the broader Peircean sense as signs whose interpretants — here the resultative action signs_-are made explicit.

3. Hoffmeyer has introduced the term semiotic scaffolding in order to characterize the role of sign processes: each step in ontogeny is temporarily supported by a web of internal sign processes assuming the correct direction of the process. A similar supportive semiotic interaction structure (at the ecological level) may play a role in phylogeny.

4. The form of a process is not its actual existence but its participation in a general class of processes, and this class can reciprocally be defined with respect to a common functional consequence achieved.

5. "That which is communicated from the Object through the Sign to the Interpretant is a Form; that is to say, it is nothing like an existent, but is a power, is the fact that something would happen under certain conditions" (Peirce, MS 793: 1-3; cf. EP2, 544, n. 22).

6. This is what Howard Pattee has pointed out many times (e.g., Pattee 2007) We think that Pattee shares with us the aim of seeing the distinction between a sign vehicle and its dynamics as a product of evolution and not simply as a taken-for-granted primitive irreducible distinction between matter and symbol. The mature Peircean notion of a sign (and Peirce's developmental taxonomies of inclusive, specialized, and degenerative types of sign aspects) is probably a more fruitful point of departure than commonsense or linguist or computer science notions of symbols.

\section{Acknowledgments}

Support from the Centre of Excellence in Cultural Theory (University of Tartu) and ETF6669 is gratefully acknowledged. 


\section{References}

Anderson M, Deely J, Krampen M, Ransdell J, Sebeok TA, Uexküll T (1984) A semiotic perspective on the sciences: Steps toward a new paradigm. Semiotica 52: 7-47.

Deacon T (1997) The Symbolic Species. London: Penguin.

Deacon T (2006a) Reciprocal linkage between self-organizing processes is sufficient for self-reproduction and evolvability. Biological Theory 1(2): $136-149$.

Deacon T (2006b) Emergence: The hole at the wheel's hub. In: The ReEmergence of Emergence (Clayton P, Davies P, eds), 111-150. Oxford: Oxford University Press.

Emmeche C (2002) The chicken and the Orphean egg: On the function of meaning and the meaning of function. Sign Systems Studies 30: 15-32.

Emmeche C, Kull K, Stjernfelt F (2002) A biosemiotic building: 13 theses. In: Reading Hoffmeyer, Rethinking Biology (Emmeche C, Kull K, Stjernfelt F), 13-24. Tartu, Estonia: Tartu University Press.

Favareau D (2007) The evolutionary history of biosemiotics. In: Introduction to Biosemiotics: The New Biological Synthesis (Barbieri M, ed), 1-67. Dordrecht: Springer.

Hoffmeyer J (1996) Signs of Meaning in the Universe. Bloomington, IN: Indiana University Press.

Hoffmeyer J (1997) Biosemiotics: Towards a new synthesis in biology. European Journal for Semiotic Studies 9: 355-376.

Hoffmeyer J (2008) Biosemiotics: An Examination into the Signs of Life and the Life of Signs. Scranton. PA: Scranton University Press.

Kant I (1952) The Critique of Judgement (Meredith JC, trans.), Oxford: Oxford University Press. Originally published in 1790 by Kritik der Urteilskraft.

Kull K (1999) Biosemiotics in the twentieth century: A view from biology. Semiotica 127: 385-414.
Kull K, ed (2001) Jakob von Uexküll: A paradigm for biology and semiotics. Semiotica 134(1-4): 1-828.

Kull K, Emmeche C, Favareau D (2008) Biosemiotic questions. Biosemiotics 1: 41-55.

Pattee HH (2007) The necessity of biosemiotics: Matter-symbol complementarity. In: Introduction to Biosemiotics: The New Biological Synthesis (Barbieri M, ed.), 115-132. Dordrecht: Springer.

Peirce CS (1931-1935, 1958) The Collected Papers of Charles Sanders Peirce. Vols. I-VI (Hartshorne C, Weiss P, eds); Vols. VII-VIII (Burks AW, ed). Cambridge, MA: Harvard University Press.

Peirce CS (1998, originally 1893-1913) The Essential Peirce: Selected Philosophical Writings, Vol. II (Peirce Edition Project, ed). Bloomington, IN: Indiana University Press. [Here referred to as EP2, followed by page number.]

Rothschild FS (1962) Laws of symbolic mediation in the dynamics of self and personality. Annals of New York Academy of Sciences 96: 774-784.

Sebeok TA (1996) Signs, bridges, origins. In: Origins of Language (Trabant J, ed), 89-115. Budapest, Hungary: Collegium Budapest.

Sebeok TA (2001) Biosemiotics: Its roots, proliferation, and prospects. Semiotica 134: 61-78.

Sebeok TA, Umiker-Sebeok J, eds (1992) Biosemiotics: The Semiotic Web 1991. Berlin: Mouton de Gruyter.

Stjernfelt F (2002) Tractatus Hoffmeyerensis: Biosemiotics as expressed in 22 basic hypotheses. Sign Systems Studies 30: 337-345.

Stjernfelt F (2007) Diagrammatology: An Investigation on the Borderlines of Phenomenology, Ontology, and Semiotics. Dordrecht: Springer.

Uexküll J (1921) Umwelt und Innenwelt der Tiere. 2nd ed. Berlin: Julius Springer.

Uexküll J (1982) The theory of meaning. Semiotica 42: 25-82. Originally published in 1940 . 